UWThPh-2001-47

November 2001

\title{
A Geometric Picture of Entanglement and Bell Inequalities
}

\author{
R.A. Bertlmann, H. Narnhofer and W. Thirring \\ Institut für Theoretische Physik \\ Universität Wien \\ Boltzmanngasse 5, A-1090 Wien
}

\begin{abstract}
We work in the real Hilbert space $\mathcal{H}_{s}$ of hermitian Hilbert-Schmidt operators and show that the entanglement witness which shows the maximal violation of a generalized Bell inequality (GBI) is a tangent functional to the convex set $S \subset \mathcal{H}_{s}$ of separable states. This violation equals the euclidean distance in $\mathcal{H}_{s}$ of the entangled state to $S$ and thus entanglement, GBI and tangent functional are only different aspects of the same geometric picture. This is explicitly illustrated in the example of two spins, where also a comparison with familiar Bell inequalities is presented.
\end{abstract}

PACS numbers: 03.67.Hk, 03.65.Bz, 03.65.Ca

Keywords: entanglement, Bell inequalities, nonlocality, tangent functional, geometry 


\section{INTRODUCTION}

The importance of entanglement [1,2] of quantum states became quite evident in the last ten years. It is the basis for such physics, like quantum cryptography [3 [6] and quantum teleportation [7, \$], and it triggered a new technology: quantum information [9.10. Entangled states lead to a violation of Bell inequalities (BI) which distinguish quantum mechanics from (all) local realistic theories [11]. Much effort has been made in studying the mathematical structure of entanglement, especially the quantification of entanglement (see, for instance, Refs. [12,13]). There exist different kinds of measures of entanglement indicating somehow the difference between entangled and separable states, which is usually related to the entropy of the states (see, e.g., Refs. [14 19]). In this paper we define a simple and quite natural measure for entanglement, a distance of certain vectors in Hilbert space which has as elements both observables and states, and we relate it to the maximum violation of a generalized Bell inequality (GBI). We work with a bipartite system in a finite-dimensional Hilbert space but generalizations are possible.

The Hilbert-Schmidt distance $D$ of a state to the set of separable states has previously been proposed as a measure of entanglement 20,21]. Our point is that if one admits all of $\mathcal{B}\left(\mathcal{H}_{A} \otimes \mathcal{H}_{B}\right)$ as entanglement witnesses then the maximal violation $B$ of the corresponding GBI equals the distance $D$ numerically. Since $D$ can be written as a minimum and $B$ as a maximum upper and lower bounds are readily available. In fact, in some standard examples one can make them coincide and thus calculate $B=D$ exactly.

Though distinct from the entropic entanglement descriptions, the Hilbert-Schmidt distance $D$ as a quantitative description of entanglement is insofar reasonable, as considered as functional of the state it is convex and invariant under local unitary transformations. This implies that states more mixed in the sense of Uhlmann 22] have a lower entanglement. However, $D$ is not monotonic decreasing under arbitrary completely positive maps in $\mathcal{H}_{A}$ or $\mathcal{H}_{B}$ but only if they have norm one. Thus whether they satisfy monotonicity in "local operations and classical communication" depends on the exact definition of this term.

We consider a finite-dimensional Hilbert space $\mathcal{H}=\mathbf{C}^{N}$, where observables $A$ are represented by all Hermitian matrices and states $w$ by density matrices. It is useful to regard these quantities as elements of a real Hilbert space $\mathcal{H}_{s}=\mathbf{R}^{N^{2}}$ with scalar product

$$
(w \mid A)=\operatorname{Tr} w A
$$

and corresponding norm

$$
\|A\|_{2}=\left(\operatorname{Tr} A^{2}\right)^{1 / 2}
$$

(we identify quantities with their representatives in $\mathcal{H}$ ). Both density matrices and observables are represented by vectors in $\mathcal{H}_{s}$, a density matrix is positive and has trace unity.

Unitary operators $U$ in $\mathcal{H}$ induce via $U A U^{*}=O A$ orthogonal operators $O$ in $\mathcal{H}_{s}$, but the homomorphism $U \rightarrow O$ is neither injective nor surjective. 


\section{SPIN EXAMPLES}

Let us begin with two examples which will be of our interest.

Example I: One spin.

Generally an observable can be written as

$$
A=\alpha \mathbf{1}+\vec{a} \cdot \vec{\sigma}, \quad \text { with } \quad \alpha \in \mathbf{R}, \vec{a} \in \mathbf{R}^{3} .
$$

The operator $A$ is a density matrix iff $\alpha=1 / 2$ and $\|\vec{a}\| \leq 1 / 2$, it gives a pure state iff $\|\vec{a}\|=1 / 2$ or $A^{2}=A$. If the state is

$$
w=\frac{1}{2}(\mathbf{1}+\vec{w} \cdot \vec{\sigma})
$$

the expectation value of $A$ is

$$
(w \mid A)=\alpha+\vec{a} \cdot \vec{w} .
$$

For us the important structural element is a tensor product $\mathcal{H}=\mathcal{H}_{A} \otimes \mathcal{H}_{B}$ which defines the set $S$ of separable (classically correlated) states $\rho_{A}^{i}, \rho_{B}^{j}$

$$
S=\left\{\rho=\sum_{i, j} c_{i j} \rho_{A}^{i} \otimes \rho_{B}^{j} \mid 0 \leq c_{i j} \leq 1, \sum_{i, j} c_{i j}=1\right\}
$$

Example II: Two spins $\vec{\sigma}_{A}$ and $\vec{\sigma}_{B}$, "Alice and Bob".

An observable $A$ can be represented by

$$
\begin{gathered}
A=\alpha \mathbf{1}+a_{i} \sigma_{A}^{i} \otimes \mathbf{1}_{B}+b_{i} \mathbf{1}_{A} \otimes \sigma_{B}^{i}+c_{i j} \sigma_{A}^{i} \otimes \sigma_{B}^{j}, \\
\frac{1}{4}\|A\|_{2}^{2}=\alpha^{2}+\sum_{i}\left(a_{i}^{2}+b_{i}^{2}\right)+\sum_{i, j} c_{i j}^{2} .
\end{gathered}
$$

Note that $c_{i j}$ can be diagonalized by 2 independent orthogonal transformations on $\sigma_{A}^{i}$ and $\sigma_{B}^{j}$ [23]. The operator $A$ is a density matrix if $\alpha=1 / 4$ and the operator norm \|\|$_{\infty}$ of $A-1 / 4$ is $\leq 1 / 4$. Since \|\|$_{2} \geq\|\|_{\infty}$ this is satisfied if

$$
\sum_{i}\left(a_{i}^{2}+b_{i}^{2}\right)+\sum_{i, j} c_{i j}^{2} \leq 1 / 16
$$

For pure states \|\|$_{2}=\|\|_{\infty}$ and $\|\rho\|_{2}=1$ is necessary and sufficient for purity. A pure separable state has the form

$$
\rho=\frac{1}{4}\left(\mathbf{1}+n_{i} \sigma_{A}^{i} \otimes \mathbf{1}_{B}+m_{i} \mathbf{1}_{A} \otimes \sigma_{B}^{i}+n_{i} m_{j} \sigma_{A}^{i} \otimes \sigma_{B}^{j}\right),
$$

with $\vec{n}^{2}=\vec{m}^{2}=1$, and gives the expectation value of $A$

$$
(\rho \mid A)=\alpha+\vec{n} \cdot \vec{a}+\vec{m} \cdot \vec{b}+n_{i} m_{j} c_{i j} .
$$




\section{GENERALIZED BELL INEQUALITY}

States which are not separable are called entangled $w \in S^{c}$, the complement in the set of states. We introduce as a measure of entanglement $D(w)$ the $\mathcal{H}_{s}$-distance of $w$ to the set $S$ of separable states

$$
D(w)=\min _{\rho \in S}\|\rho-w\|_{2} .
$$

Since

$$
\|\rho-w\|_{2}^{2}=\operatorname{Tr}\left(\rho^{2}+\omega^{2}-2 \sqrt{\rho} \omega \sqrt{\rho}\right) \leq \operatorname{Tr}\left(\rho^{2}+\omega^{2}\right) \leq 2
$$

we generally have

$$
0 \leq D(w) \leq \sqrt{2} .
$$

Usually the Bell inequality refers to an operator in the tensor product where by classical arguments only some range of expectation values can be expected whereas quantum mechanics permits other values. A Bell inequality in a generalized sense is given by an observable $A \nsupseteq 0$ for which

$$
(\rho \mid A) \geq 0 \quad \forall \rho \in S .
$$

Thus $\exists w$ such that

$$
(w \mid A)<0 \quad \text { for some } \quad w \in S^{c} .
$$

Such elements $A \in \mathcal{A}_{W}$ are called entanglement witnesses [24,25]. A product operator can never be $\in \mathcal{A}_{W}$ but already the sum of two products serves for the CHSH (Clauser, Horne, Shimony, Holt) inequality [26]. But the number of summands is not restricted in $\mathcal{A}_{W}$. The operator $A \in \mathcal{A}_{t}$ becomes a tangent functional if in addition $\exists \rho_{0} \in S$ such that $\left(\rho_{0} \mid A\right)=0$. Since $S$ is a convex subset of the state space such tangential $A$ 's always exist. Even more, the set $S$ is characterized by the tangent functionals and the $\rho_{0}$ 's with $\left(\rho_{0} \mid A\right)=0$, for some $A \in \mathcal{A}_{t}$, are the boundary $\partial S$ of $S$.

Frequently a bigger set than $S$ is considered as classically explainable in a local hidden variable theory. Bell inequalities are those which contradict even those sets. To avoid misunderstandings we call generalized Bell inequalities expectation values which contradict the predictions from $S$, the set of separable states.

Thus the GBI (3.3) is violated by an entangled state $w$, Eq.(3.4), and we get the following inequality for some $A \in \mathcal{A}_{W}$

$$
(\rho \mid A)>(w \mid A) \quad \forall \rho \in S .
$$

Considering now the maximal violation of the GBI

$$
B(w)=\max _{\|A-\alpha\|_{2} \leq 1}\left[\min _{\rho \in S}(\rho \mid A)-(w \mid A)\right],
$$


we find the following result

\section{Theorem:}

i) The maximal violation of the GBI is equal to the distance of $w$ to the set $S$

$$
B(w)=D(w) \quad \forall w
$$

ii) The min of $D$ is attained for some $\rho_{0}$ and the max of $B$ for

$$
A_{\max }=\frac{\rho_{0}-w-\left(\rho_{0} \mid \rho_{0}-w\right) \mathbf{1}}{\left\|\rho_{0}-w\right\|_{2}} \in \mathcal{A}_{t} .
$$

iii) For $B=D$ the following two-sided variational principle holds

$$
\min _{\rho \in S}\left(\rho-w \mid \frac{\rho^{\prime}-w}{\left\|\rho^{\prime}-w\right\|_{2}}\right) \leq B(w) \leq\left\|\rho^{\prime}-w\right\|_{2} \quad \forall \rho^{\prime} \in S .
$$

(For an illustration, see Fig. 1; for a similar view, particularly about $A_{\max }$, see Ref. [27]).

\section{Remark:}

The proof of the Theorem does not use the product structure of the Hilbert space $\mathcal{H}$ but only the geometric properties of the Euclidean distance in $\mathcal{H}_{s}$. It can be illustrated already with one spin where the set of separable states $S$ is replaced by $S_{z}$

$$
S_{z}=\left\{\rho=\frac{1}{2}\left(\mathbf{1}+\lambda \sigma_{z}\right),|\lambda| \leq 1\right\}
$$

and

$$
w=\frac{1}{2}(\mathbf{1}+\vec{w} \cdot \vec{\sigma}), \quad\|w\|_{2} \leq 1
$$

is considered as the analogue of an entangled state, if $w_{x}$ or $w_{y} \neq 0$.

The observables $A$ with $\|A\|_{2}=1$ are of the form

$$
A=\frac{\alpha \mathbf{1}+\vec{a} \cdot \vec{\sigma}}{\sqrt{2}\left(\alpha^{2}+a^{2}\right)^{1 / 2}}, \quad \text { and } \quad a=\|\vec{a}\|, \vec{a} \in \mathbf{R}^{3} .
$$

For the $\mathcal{H}_{s}$-distance $D$, our measure of entanglement, we calculate

$$
\min _{\rho}\|\rho-w\|_{2}^{2}=\min _{\lambda} \frac{1}{4}\left\|\lambda \sigma_{z}-\vec{w} \cdot \vec{\sigma}\right\|_{2}^{2}=\min _{\lambda} \frac{1}{2}\left(\left(\lambda-w_{z}\right)^{2}+w_{x}^{2}+w_{y}^{2}\right)=\frac{1}{2}\left(w_{x}^{2}+w_{y}^{2}\right)
$$

attained for $\lambda=w_{z}$, so that we have

$$
D(w)=\frac{1}{\sqrt{2}}\left(w_{x}^{2}+w_{y}^{2}\right)^{1 / 2}
$$




\section{FIGURES}

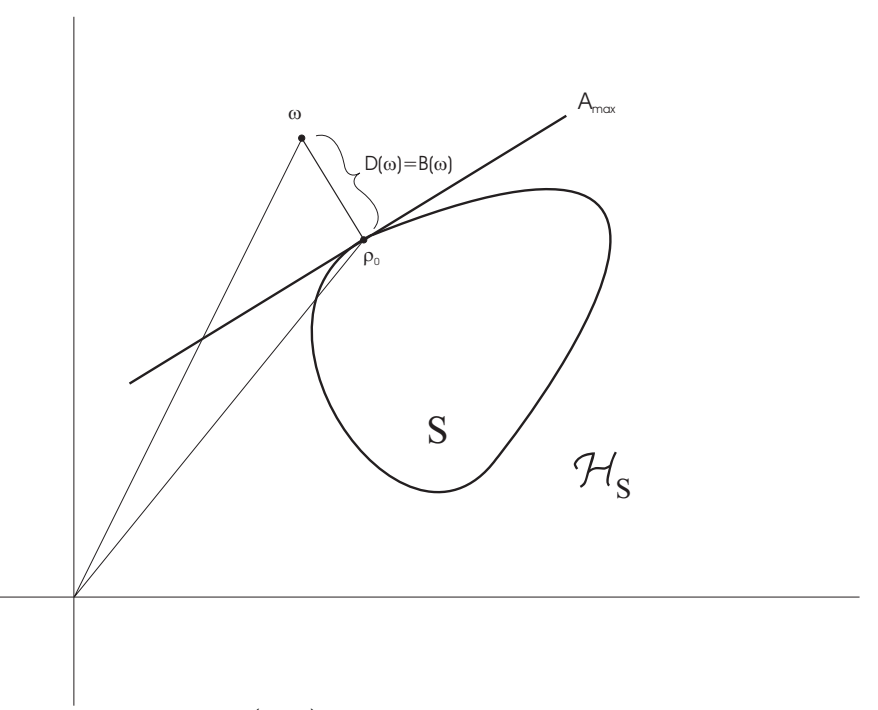

FIG. 1. Illustration of Theorem (3.7). The maximal violation of GBI $B(w)$, Eq.(3.6), which is equal to the $\mathcal{H}_{s}$-distance $D(w)$, Eq.(3.1), of an entangled state $w$ to the set $S$ of separable states, is shown together with the tangent plane defined by $A_{\max }$ (3.8).

On the other hand, we find for the maximal violation of the GBI

$$
\begin{aligned}
B(w) & =\max _{\vec{a}, \alpha} \min _{\lambda} \frac{1}{2}\left(\lambda \sigma_{z}-\vec{w} \cdot \vec{\sigma} \mid \frac{\alpha \mathbf{1}+\vec{a} \cdot \vec{\sigma}}{\sqrt{2}\left(\alpha^{2}+a^{2}\right)^{1 / 2}}\right)=\max _{\vec{a}, \alpha} \frac{-1}{\sqrt{2}} \cdot \frac{\left|a_{z}\right|+\vec{w} \cdot \vec{a}}{\left(\alpha^{2}+a^{2}\right)^{1 / 2}} \\
& =\frac{1}{\sqrt{2}}\left(w_{x}^{2}+w_{y}^{2}\right)^{1 / 2} .
\end{aligned}
$$

Here the observable

$$
A_{\max }=-\frac{w_{x} \sigma_{x}+w_{y} \sigma_{y}}{\sqrt{2}\left(w_{x}^{2}+w_{y}^{2}\right)^{1 / 2}}
$$

is the tangent functional $\forall \rho \in S_{z}, \partial S_{z}=S_{z}$.

Note that for the maximal violation of the GBI (3.14) the $\min _{\rho \in S}$ is attained for $\frac{1}{2}\left(1-\sigma_{z}\right)$ if $a_{z}>0$ and not for $\frac{1}{2}\left(1+w_{z} \sigma_{z}\right)$ as in case of the distance (3.13). It means that for $D$ the $\min _{\rho}$ is not necessarily attained for a pure state but for $B$ it is since it is effectively a max. Thus the equality $B=D$, Theorem part (3.7), is not so trivial since the extrema may be attained at disjointed sets. Then min max may be bigger than max min as can be seen already in $\min _{i}$ and $\max _{j}$ for the matrix

$$
M_{i j}=\left(\begin{array}{ll}
0 & 1 \\
1 & 0
\end{array}\right) .
$$


Proof of the Theorem: Eq.(3.7)

$D(w)=\min _{\rho \in S}\|\rho-w\|_{2}$ is attained for some $\rho_{0}$ since \|\|$_{2}$ is continuous and $S$ is compact. Now take for $A-\alpha=\left(\rho_{0}-w\right) /\left\|\rho_{0}-w\right\|_{2}$ in the definition of $B$ and use the orthogonal decomposition with respect to this unit vector, $\mathcal{H}_{s} \ni v=v_{\|}+v_{\perp},\left(v_{\perp} \mid \rho_{0}-w\right)=0$. Therefore we can apply simple Euclidean geometry and decompose the vector $\rho-w$ in the above sense.

We also remember that $\rho_{0}-w$ is the normal to the tangent plane to $S$, which means

$$
\left\|(\rho-w)_{\|}\right\|_{2} \geq\left\|\left(\rho_{0}-w\right)_{\|}\right\|_{2}=\left\|\rho_{0}-w\right\|_{2}
$$

since $S$ is convex, see Fig. 2. This we can prove in the following way. The tangent $A_{\max }$ divides the state space into $\mathcal{H}_{w}=\left\{\rho:\left\|(\rho-w)_{\|}\right\|_{2}<\left\|\left(\rho_{0}-w\right)_{\|}\right\|_{2}\right\}$, which contains $w$, and $\mathcal{H}_{w}^{c}$, the complement to $\mathcal{H}_{w}$. If $\mathcal{H}_{w}$ were to contain $\rho \in S$ then because of the convexity of $S$ it would contain all $\rho_{\lambda}=(1-\lambda) \rho_{0}+\lambda \rho, \quad \lambda \in[0,1]$. Since $\rho_{\lambda}$ would have an angle of less than $90^{\circ}$ with $\rho_{0}-w$ there would be a $\rho_{\lambda}$ inside the ball $\|(\rho-w)\|_{2}<\left\|\left(\rho_{0}-w\right)\right\|_{2}=D(w)$ and $\rho_{0}$ would not be the point of $S$ of minimal distance to $w$. Therefore $S \subset \mathcal{H}_{w}^{c}$ and $\left\|(\rho-w)_{\|}\right\|_{2} \geq\left\|\left(\rho_{0}-w\right)\right\|_{2} \quad \forall \rho \in S$.

Using above arguments we obtain

$$
\begin{gathered}
B(w) \geq \min _{\rho}\left(\rho-w \mid \frac{\rho_{0}-w}{\left\|\rho_{0}-w\right\|_{2}}\right) \geq \min _{\rho}\left((\rho-w)_{\|} \mid \frac{\rho_{0}-w}{\left\|\rho_{0}-w\right\|_{2}}\right) \\
\geq\left(\rho_{0}-w \mid \frac{\rho_{0}-w}{\left\|\rho_{0}-w\right\|_{2}}\right)=\left\|\rho_{0}-w\right\|_{2}=D(w) .
\end{gathered}
$$

On the other hand, $D$ and $B$ can be written as $\min _{\rho} \max _{A}$ and $\max _{A} \min _{\rho}$ of $(\rho-w \mid A)$ and generally we have $\min \max \geq \max \min$. So a priori we know $D(w) \geq B(w)$ and we conclude $D(w)=B(w)$.

\section{PROPERTIES OF THE GENERALIZED BELL INEQUALITY}

Now we discuss the properties of $D(w)$, Eq.(3.1), the $\mathcal{H}_{s}$-distance of $w$ to the set $S$ of separable states, which is equal to $B(w)$, Eq.(3.6), the maximal violation of the GBI.

Properties of $D(w)$ :

i) $D(w)$ is convex,

ii) $D(w)$ is continuous,

iii) $D(w)=D\left(U_{A} \otimes U_{B} w U_{A}^{*} \otimes U_{B}^{*}\right) \quad \forall$ unitary operators $U_{A, B}$.

iv) $D(w)$ is monotonic decreasing under mixing enhancing maps, see e.g. Ref. [28. 


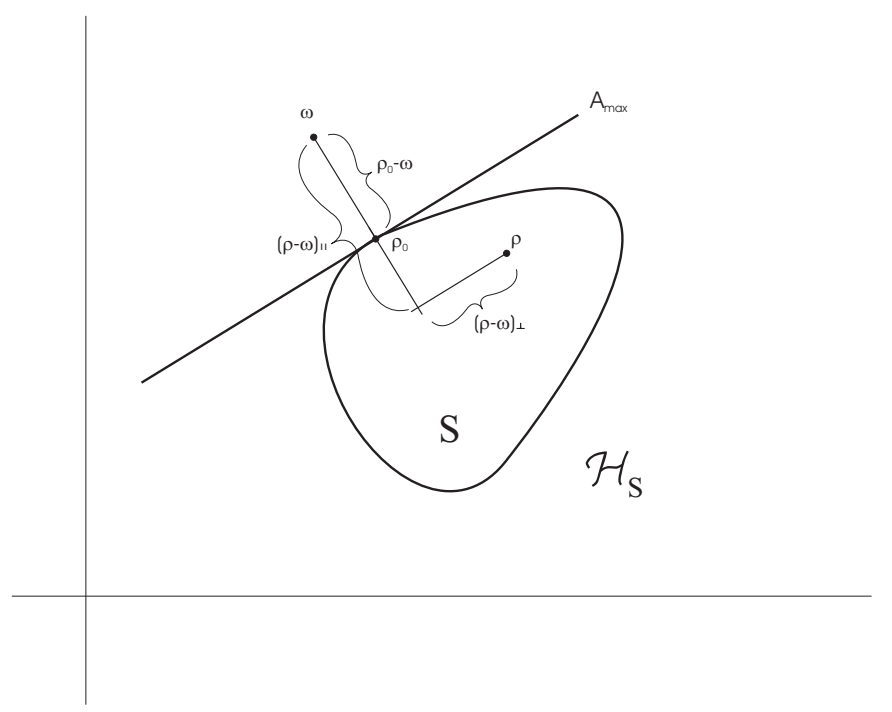

FIG. 2. For illustration we have drawn the vectors used in the Proof of Theorem (3.7).

\section{Remarks:}

ad i) It means that by mixing the entanglement decreases and the maximally entangled states must be pure. This is to be expected since the tracial state $w_{\mathrm{tr}}=$ $1 /\left(\operatorname{dim} \mathcal{H}_{A} \operatorname{dim} \mathcal{H}_{B}\right)$ is separable $\Leftrightarrow D(w)=0$.

Furthermore the set $\{w \mid D(w)<c\}$ is convex.

ad ii) It tells us for an entangled state a neighborhood of it is also entangled. Actually a neighborhood of the tracial state is also separable.

ad iii) The state space decomposes into equivalence classes of states with the same entanglement. All pure separable states are in the same equivalence class.

ad iv) Mixing enhancing maps are essentially a combination of unitary transformations and convex combinations.

\section{Proofs of the $\mathrm{B}, \mathrm{D}$ properties:}

i) $B(w)$ and $D(w)$ are continuous:

$$
\begin{aligned}
& |(w+\delta-\rho \mid A)-(w-\rho \mid A)| \leq \varepsilon \quad \forall\|\delta\|_{2} \leq \varepsilon,\|A\|_{2} \leq 1 \\
& \Rightarrow \quad \mid(B \text { or } D)(w+\delta)-(B \text { or } D)(w) \mid \leq \varepsilon \quad \forall\|\delta\|_{2} \leq \varepsilon .
\end{aligned}
$$

ii) $B(w)$ is convex:

$$
\begin{aligned}
& B\left(\sum_{i} \lambda_{i} w_{i}\right)=\max _{A} \sum_{i} \lambda_{i}\left(\min _{\rho \in S}(\rho \mid A)-\left(w_{i} \mid A\right)\right) \\
\leq & \sum_{i} \lambda_{i}\left(\max _{A}\left[\min _{\rho \in S}(\rho \mid A)-\left(w_{i} \mid A\right)\right]\right)=\sum_{i} \lambda_{i} B\left(w_{i}\right) .
\end{aligned}
$$


iii) $D(w)=D\left(U_{A} \otimes U_{B} w U_{A}^{*} \otimes U_{B}^{*}\right)$ follows from the invariance of $S$ under $U_{A} \otimes U_{B}$.

iv) The monotonic decrease under mixing enhancing maps is a consequence of points i) and iii).

The "most" separable state $w_{\mathrm{tr}}=1 / \operatorname{dim} \mathcal{H}$ is a convex combination of most entangled states. From the properties of $D(w)$ we get the following artistic impression. In the state space there is a plane around $w_{\text {tr }}$ with $D(w)=0$. From it emerge valleys with $D(w)=0$

to the pure separable states on the boundary. In their neighborhood are entangled states thus $D$ slopes up in such a way that the regions $D \leq c$, with $0 \leq c \leq D_{\max }$, are convex. On the boundary of the state space also sit the states with $D=D_{\max }$ and they form a rim. Since $U_{A} \otimes U_{B}$ act continuously in a neighborhood of maximally entangled states there are others with $D=D_{\max }$ but also some with $D<D_{\max }$ which one gets by mixing in a little bit with the separable states.

This somewhat poetic description is mathematically supplemented by considering $S$ as a subset of the state space $S \cup S^{c} \subset \mathcal{H}_{s}$, so the boundary $\partial S$ are those elements of $S$ where in each neighborhood there are entangled states.

\section{GEOMETRY OF SEPARABLE STATES}

What is the geometric structure of the set $S$ of separable states? Let us investigate its properties.

\section{Properties of S:}

i) The dimensions of both $S$ and $S^{c}$ are $N^{2}-1$.

ii) Pure separable states belong to the boundary $\partial S$ and convex combinations of two of them are still on $\partial S$.

iii) If a mixture $\rho=\sum_{i=1}^{n} \mu_{i} \rho_{i}$ is on $\partial S$ then there is a face, i.e.

$$
\bar{\rho}=\sum_{i=1}^{n} \bar{\mu}_{i} \rho_{i} \in \partial S \quad \forall \bar{\mu}_{i} \geq 0, \sum_{i=1}^{n} \bar{\mu}_{i}=1 .
$$

iv) If $\mathcal{H}_{A}=\mathcal{H}_{B}\left(=\mathbf{C}^{\sqrt{N}}\right)$ then $\partial S$ contains at least $N$ dimensional faces.

v) $S$ is invariant under $T_{A} \otimes \mathbf{1}_{B}$, with $T_{A}$ any positive map $\mathcal{B}\left(\mathcal{H}_{A}\right) \rightarrow \mathcal{B}\left(\mathcal{H}_{A}\right)$.

vi) If $A \nsupseteq 0$ but $\left(T_{A} \otimes \mathbf{1}_{B}\right) A \geq 0$ then $A \in \mathcal{A}_{W}$ and if $\exists \rho_{0} \in S$ such that $\left(\rho_{0} \mid A\right)=0$ then $A \in \mathcal{A}_{t}$. 


\section{Remarks:}

ad i) It means that both $S$ and $S^{c}$ are everywhere thick and do not have pieces of lower dimensions.

ad ii) Clearly the convex combination of two pure states lies (for $N>2$ ) on the boundary of the state space since in each neighborhood there are not positive functionals. Here we have the stronger statement that in each neighborhood there are entangled states.

ad iii) If $\partial S$ has a n-dimensional flat part this means that mixtures of $n$ pure states are on $\partial S$. Point iii) affirms the converse in the sense that in the decomposition the $\rho_{i}$ 's span a face.

ad iv) It says that $n=N$ actually occurs.

ad v) Strangely, the tensor product of two positive maps is not necessarily positive but applied to separable states it is.

\section{Proofs of the properties of $S$ :}

i) $S$ has the full dimension of $N$ since a neighborhood of the tracial state $w_{\mathrm{tr}}=1 / N$ is separable and as a convex set it has everywhere the same dimension. The complement $S^{c}$, the set of entangled states, has the full dimension since $D$ is continuous and if $D(w)>0$ it is so for a neighborhood of $w$.

ii) $\rho$ is pure and separable $\Rightarrow \rho \in \partial S$ :

If $\rho=|\phi \otimes \psi\rangle\langle\phi \otimes \psi|$ (pure and separable) then $\left|\phi \otimes \psi+\varepsilon \phi^{\prime} \otimes \psi^{\prime}\right\rangle\left\langle\phi \otimes \psi+\varepsilon \phi^{\prime} \otimes \psi^{\prime}\right|$ comes for $\varepsilon \rightarrow 0$ arbitrarily close and is $\forall \varepsilon$ pure and not a product state $\Rightarrow$ it is entangled $\Rightarrow \rho \in \partial S$.

$\rho_{i}$ is pure and separable $\Rightarrow \rho_{\lambda}=\lambda \rho_{1}+(1-\lambda) \rho_{2} \in \partial S$ :

Let us take $\rho_{i}=\left|\phi_{i} \otimes \psi_{i}\right\rangle\left\langle\phi_{i} \otimes \psi_{i}\right|$ and consider

$\lambda\left|\phi_{1} \otimes \psi_{1}+\varepsilon \phi_{2} \otimes \psi_{2}\right\rangle\left\langle\phi_{1} \otimes \psi_{1}+\varepsilon \phi_{2} \otimes \psi_{2}|+(1-\lambda)| \phi_{2} \otimes \psi_{2}+\varepsilon^{\prime} \phi_{1} \otimes \psi_{1}\right\rangle\left\langle\phi_{2} \otimes \psi_{2}+\varepsilon^{\prime} \phi_{1} \otimes \psi_{1}\right|$.

For $\varepsilon, \varepsilon^{\prime} \rightarrow 0$ it comes arbitrarily close to $\rho_{\lambda}$ but in the two dimensional Hilbert subspace spanned by $\phi_{i} \otimes \psi_{i}(i=1,2)$ the only separable pure states are of the form $\rho_{1,2}$. Thus a state that is not a linear combination of $\rho_{1}$ and $\rho_{2}$ needs for its decomposition into pure states at least one pure entangled state, and is therefore entangled itself. Therefore we have an entangled state arbitrarily close to $\rho_{\lambda} \Rightarrow$ $\rho_{\lambda} \in \partial S$ (compare with Refs. 229,30]).

iii) For a tangent functional $A$ at $\rho=\sum \mu_{i} \rho_{i}, \rho_{i} \in S$, we have

$$
\begin{gathered}
0=(\rho \mid A)=\sum \mu_{i}\left(\rho_{i} \mid A\right) \quad \Rightarrow \quad\left(\rho_{i} \mid A\right)=0 \quad \forall i \\
\Rightarrow \quad\left(\sum \bar{\mu}_{i} \rho_{i} \mid A\right)=0 \quad \Rightarrow \quad \sum \bar{\mu}_{i} \rho_{i} \in \partial S .
\end{gathered}
$$


iv) For a given tangent functional $A_{t}=A_{1}-A_{2}, A_{i} \geq 0,\left\|A_{2}\right\|_{2}=1$ there exists an entangled state $w$ with $\left(w \mid A_{t}\right)=-\left(w \mid A_{2}\right) \leq-1+\varepsilon$. The homotopic state $\bar{w}=$ $\left(1-\frac{\varepsilon}{2}\right) w+\frac{\varepsilon}{2} w_{\text {tr }}$ is also entangled since $D(\bar{w})$ is continuous, and the corresponding density matrix is invertible and needs $N$ components to be decomposed into pure states. There exists a continuous path from the entangled $\bar{w}$ to the separable $w_{\text {tr }}$ formed from states with corresponding invertible density matrices. When this path passes the boundary $\partial S$ then according to property iii) we obtain a separable state embedded in a N-dimensional face of $\partial S$.

v) Follows from the results in Ref. 24.

vi) Follows from v) and the definitions of $\mathcal{A}_{W}$ and $\mathcal{A}_{t}$.

\section{GEOMETRY OF ENTANGLED AND SEPARABLE STATES OF SPIN SYSTEMS}

We focus again on the two spin example and calculate the entanglement of the following quantum states.

Example: Alice and Bob, the "Werner states".

Let us consider Werner states [31] which can be parameterized by

$$
w_{\alpha}=\frac{1-\alpha \vec{\sigma}_{A} \otimes \vec{\sigma}_{B}}{4}
$$

and they are possible density matrices for $-1 / 3 \leq \alpha \leq 1$ since $\vec{\sigma}_{A} \otimes \vec{\sigma}_{B}$ has the eigenvalues $-3,1,1,1$. To calculate the entanglement we first mix product states to get

$$
\frac{1}{2}\left\{\frac{\left(\mathbf{1}_{A}-\sigma_{A}^{x}\right) \otimes\left(\mathbf{1}_{B}+\sigma_{B}^{x}\right)}{2}+\frac{\left(\mathbf{1}_{A}+\sigma_{A}^{x}\right) \otimes\left(\mathbf{1}_{B}-\sigma_{B}^{x}\right)}{2}\right\}=\frac{1-\sigma_{A}^{x} \otimes \sigma_{B}^{x}}{4}
$$

and then with $x \rightarrow y, x \rightarrow z$ finally

$$
\rho_{0}=\frac{1}{4}\left(\mathbf{1}-\frac{1}{3} \vec{\sigma}_{A} \otimes \vec{\sigma}_{B}\right) \in S
$$

This seems a good $\rho_{0}$ for $w_{\alpha}$ if $1 / 3<\alpha \leq 1$; and we use it for $\rho^{\prime}$ in the Theorem part iii), Eq. (3.9). With $\rho_{0}-w_{\alpha}=\frac{1}{4}(\alpha-1 / 3) \vec{\sigma}_{A} \otimes \vec{\sigma}_{B}$ and $\left\|\vec{\sigma}_{A} \otimes \vec{\sigma}_{B}\right\|_{2}=2 \sqrt{3}$ we get

$$
D\left(w_{\alpha}\right) \leq \frac{\sqrt{3}}{2}(\alpha-1 / 3)
$$

On the other hand, the observable which according to Eq.(3.8) violates the GBI (3.5) maximally is $A=-\vec{\sigma}_{A} \otimes \vec{\sigma}_{B} / 2 \sqrt{3}$. In fact, 


$$
\left(w_{\alpha} \mid-\frac{\vec{\sigma}_{A} \otimes \vec{\sigma}_{B}}{2 \sqrt{3}}\right)=\alpha \frac{\sqrt{3}}{2}
$$

and a pure product $\rho$ gives $\left(\rho \mid \vec{\sigma}_{A} \otimes \vec{\sigma}_{B}\right)=\vec{n} \cdot \vec{m}$. Since $|\vec{n} \cdot \vec{m}| \leq 1$ and this cannot be increased by mixing we have proved $B\left(w_{\alpha}\right) \geq \frac{\sqrt{3}}{2}(\alpha-1 / 3)$. But $\bar{D}$ and $B$ can be written as $\min _{\rho} \max _{A}$ and $\max _{A} \min _{\rho}$ of $(\rho-w \mid A)$ and generally min max $\geq$ max min so a priori we know $D(w) \geq B(w)$. Therefore the above inequalities imply

$$
D\left(w_{\alpha}\right)=B\left(w_{\alpha}\right)=\frac{\sqrt{3}}{2}(\alpha-1 / 3) \quad \forall 1 / 3 \leq \alpha \leq 1 .
$$

Furthermore the minimizing $\rho_{0}$ is given by Eq. $(6.2)$ and the maximizing observable is $-\vec{\sigma}_{A} \otimes \vec{\sigma}_{B} / 2 \sqrt{3}$. Considering the state with $\alpha=1$ we finally get

$$
\left(\rho \mid-\vec{\sigma}_{A} \otimes \vec{\sigma}_{B}\right) \leq 1 \quad \forall \rho \in S \quad \text { and } \quad\left(w_{\alpha=1} \mid-\vec{\sigma}_{A} \otimes \vec{\sigma}_{B}\right)=3,
$$

and the GBI is violated by a factor 3 . But this ratio is not significant since by $A \rightarrow A+c \mathbf{1}$ it can be given any value. Meaningful is $B(w)$ since it is not affected by this change.

For the parameter values $-1 / 3 \leq \alpha \leq 1 / 3$ the states $w_{\alpha}$ (6.1) are separable, for $1 / 3<\alpha<1$ they are mixed entangled, and the limit $\alpha=1$ represents the spin singlet state which is pure and maximally entangled.

Let us consider next the tangent functionals. From expression (3.8) we get our old friend, the flip operator 31

$$
A_{t}=\frac{1}{4}\left(\mathbf{1}+\vec{\sigma}_{A} \otimes \vec{\sigma}_{B}\right)
$$

It is not positive but applying the transposition operator $T$, defined by $T\left(\sigma^{i}\right)_{k l}=\left(\sigma^{i}\right)_{l k}$, on Bob it turns into a positive operator

$$
\left(\mathbf{1}_{A} \otimes T_{B}\right) A_{t}=\frac{1}{4}\left(\mathbf{1}+\sigma_{A}^{x} \otimes \sigma_{B}^{x}-\sigma_{A}^{y} \otimes \sigma_{B}^{y}+\sigma_{A}^{z} \otimes \sigma_{B}^{z}\right)
$$

which can be nicely written as $4 \times 4$ matrices

$$
A_{t}=\frac{1}{4}\left(\begin{array}{llll}
2 & 0 & 0 & 0 \\
0 & 0 & 2 & 0 \\
0 & 2 & 0 & 0 \\
0 & 0 & 0 & 2
\end{array}\right) \quad\left(\mathbf{1}_{A} \otimes T_{B}\right) A_{t}=\frac{1}{4}\left(\begin{array}{cccc}
2 & 0 & 0 & 2 \\
0 & 0 & 0 & 0 \\
0 & 0 & 0 & 0 \\
2 & 0 & 0 & 2
\end{array}\right)
$$

Operator $A_{t}$ is not only tangent functional for the mixed separable state $\rho_{0}$ (6.2) but with

$$
\begin{aligned}
\left(\rho \mid A_{t}\right) & =\frac{1}{16} \operatorname{Tr}\left[\left(\mathbf{1}+n_{i} \sigma_{A}^{i} \otimes \mathbf{1}_{B}+m_{i} \mathbf{1}_{A} \otimes \sigma_{B}^{i}+n_{i} m_{j} \sigma_{A}^{i} \otimes \sigma_{B}^{j}\right)\left(\mathbf{1}+\vec{\sigma}_{A} \otimes \vec{\sigma}_{B}\right)\right] \\
& =\frac{1}{4}(1+\vec{n} \cdot \vec{m})=0
\end{aligned}
$$


it is a tangent functional for all pure separable states with $\vec{m}=-\vec{n}$, which is especially the case for those states used for $\rho_{0}(\underline{6.2})$. This illustrates point iii) of the properties of the set $S$.

On the other hand, for the pure separable states in this face we can find other tangent functionals. For example, for the state

$$
\rho_{z}=\frac{1}{4}\left(\mathbf{1}+\sigma_{A}^{z} \otimes \mathbf{1}_{B}+\mathbf{1}_{A} \otimes \sigma_{B}^{z}+\sigma_{A}^{z} \otimes \sigma_{B}^{z}\right)
$$

we easily see within $4 \times 4$ matrices that the operators

$$
\rho_{z}=\frac{1}{4}\left(\begin{array}{cccc}
1 & 0 & 0 & 0 \\
0 & 0 & 0 & 0 \\
0 & 0 & 0 & 0 \\
0 & 0 & 0 & 0
\end{array}\right)
$$

and

$$
A_{t}=\frac{1}{a^{2}+b^{2}}\left(\begin{array}{cccc}
0 & 0 & 0 & a b \\
0 & a^{2} & 0 & 0 \\
0 & 0 & b^{2} & 0 \\
a b & 0 & 0 & 0
\end{array}\right) \quad\left(\mathbf{1}_{A} \otimes T_{B}\right) A_{t}=\frac{1}{a^{2}+b^{2}}\left(\begin{array}{cccc}
0 & 0 & 0 & 0 \\
0 & a^{2} & a b & 0 \\
0 & a b & b^{2} & 0 \\
0 & 0 & 0 & 0
\end{array}\right)>0
$$

satisfy the requirement of a tangent functional. For the state $\rho_{x}$ (let $z \rightarrow x$ in Eq.(6.11)), however, we have $\left(\rho_{x} \mid A_{t}\right)=1$.

\section{Remark:}

At this stage we would like to compare our approach to generalized Bell inequalities with the more familiar type of inequalities (compare also with Refs. [25, 32]). Usually the BI is given by an operator in the tensor product, where by classical arguments only some range of expectation values can be expected, whereas the quantum case permits an other range. In our case, classically we would expect

$$
0 \leq\left(\rho_{\text {class }} \mid \mathbf{1}+\vec{\sigma}_{A} \otimes \vec{\sigma}_{B}\right) \leq 2 \text { or } \quad\left|\left(\rho_{\text {class }} \mid \vec{\sigma}_{A} \otimes \vec{\sigma}_{B}\right)\right| \leq 1
$$

because the expectation value of the individual spin is maximally 1 and the largest (smallest) value should be obtained when they are parallel (antiparallel). This range of expectation values can exactly be achieved by all separable states $\rho \in S$, whereas we can find an entangled quantum state, the spin singlet state $w_{\alpha=1}$ (6.1), which gives

$$
\left(w_{\alpha=1} \mid \mathbf{1}+\vec{\sigma}_{A} \otimes \vec{\sigma}_{B}\right)=-2 \quad \text { or } \quad\left|\left(w_{\alpha=1} \mid \vec{\sigma}_{A} \otimes \vec{\sigma}_{B}\right)\right|=3
$$

This demonstrates that the tensor product operator $\vec{\sigma}_{A} \otimes \vec{\sigma}_{B}$ cannot be written as a $\mathrm{CHSH}$ operator, where the ratio is limited by $\sqrt{2}$. If we perturb a pure separable state like

$$
\rho_{\varepsilon}=\frac{1}{4}\left(\mathbf{1}+n_{i} \sigma_{A}^{i} \otimes \mathbf{1}_{B}-n_{i} \mathbf{1}_{A} \otimes \sigma_{B}^{i}-\left(n_{i} n_{j}+\varepsilon_{i j}\right) \sigma_{A}^{i} \otimes \sigma_{B}^{j}\right)
$$


then the expectation value

$$
\left(\rho_{\varepsilon} \mid \mathbf{1}+\vec{\sigma}_{A} \otimes \vec{\sigma}_{B}\right)=\mathcal{O}(\varepsilon)
$$

is of order $\mathcal{O}(\varepsilon)$, as the operator constructed in Ref. [33], which shows the sensitivity of $\mathcal{A}_{t}(6.13)$ as entanglement witness.

In the familiar Bell inequality derived by $\mathrm{CHSH}$ [26]

$$
\left(\rho \mid A_{C H S H}\right) \leq 2
$$

with $\rho \in S$ (actually CHSH consider classical states $\rho_{\text {class }}$, a generalization of separable states, in their work [26]), a rather general observable (a 4 parameter family of observables)

$$
A_{C H S H}=\vec{a} \cdot \vec{\sigma}_{A} \otimes\left(\vec{b}-\vec{b}^{\prime}\right) \cdot \vec{\sigma}_{B}+\vec{a}^{\prime} \cdot \vec{\sigma}_{A} \otimes\left(\vec{b}+\vec{b}^{\prime}\right) \cdot \vec{\sigma}_{B}
$$

is used, where $\vec{a}, \vec{a}^{\prime}, \vec{b}, \vec{b}^{\prime}$ are any unit vectors in $\mathbf{R}^{3}$.

However, the spin singlet state $w_{\alpha=1}$ (6.1) gives

$$
\left(w_{\alpha=1} \mid A_{C H S H}\right)=-\vec{a} \cdot\left(\vec{b}-\vec{b}^{\prime}\right)-\vec{a}^{\prime} \cdot\left(\vec{b}+\vec{b}^{\prime}\right),
$$

which violates the $\mathrm{CHSH}$ inequality (6.18) maximally

$$
\left(w_{\alpha=1} \mid A_{C H S H}\right)=2 \sqrt{2}
$$

for appropriate angles: $(\vec{a}, \vec{b})=\left(\vec{a}^{\prime}, \vec{b}\right)=\left(\vec{a}^{\prime}, \vec{b}^{\prime}\right)=135^{\circ},\left(\vec{a}, \vec{b}^{\prime}\right)=45^{\circ}$, whereas in this case we find (for all separable states $\rho \in S$ )

$$
\max _{\rho \in S}\left(\rho \mid A_{C H S H}\right)=\sqrt{2} .
$$

Bell in his original work [34] considers only 3 different directions in space (which corresponds to the specific case $\vec{a}^{\prime}=-\vec{b}^{\prime}$ in CHSH (6.19)) and assumes a strict anticorrelation

$$
\left(\rho \mid \vec{a}^{\prime} \cdot \vec{\sigma}_{A} \otimes \vec{a}^{\prime} \cdot \vec{\sigma}_{B}\right)=-1
$$

Then he derives the inequality

$$
\left(\rho \mid A_{\text {Bell }}\right) \leq 1
$$

(which clearly follows from (6.18) under the mentioned conditions), where now the observable is

$$
A_{\text {Bell }}=\vec{a} \cdot \vec{\sigma}_{A} \otimes\left(\vec{b}-\vec{b}^{\prime}\right) \cdot \vec{\sigma}_{B}-\vec{b}^{\prime} \cdot \vec{\sigma}_{A} \otimes \vec{b} \cdot \vec{\sigma}_{B}
$$

The expectation value of Bell's observable in the spin singlet state 


$$
\left(w_{\alpha=1} \mid A_{\text {Bell }}\right)=-\vec{a} \cdot\left(\vec{b}-\vec{b}^{\prime}\right)+\vec{b}^{\prime} \cdot \vec{b}
$$

lies (maximally) outside the range of BI (6.24)

$$
\left(w_{\alpha=1} \mid A_{\text {Bell }}\right)=\frac{3}{2}
$$

for the angles $\left(\vec{a}, \vec{b}^{\prime}\right)=\left(\vec{b}^{\prime}, \vec{b}\right)=60^{\circ},(\vec{a}, \vec{b})=120^{\circ}$, whereas now we have for all anticorrelated separable states $\rho_{a}=\{\rho \in S \mid$ with $\vec{n} \cdot \vec{m}=-1\}$

$$
\max _{\rho_{a} \in S}\left(\rho \mid A_{\text {Bell }}\right)=\frac{3}{4} \text {. }
$$

Note that generally $\forall \rho \in S$ the maximum (6.28) is larger, namely $\sqrt{3} / 2$ instead of $3 / 4$.

We observe that the maximal violation of the GBI, Eq. (3.6), is largest for our observable $-\vec{\sigma}_{A} \otimes \vec{\sigma}_{B}$, where the difference between singlet state and separable state is 2 (recall Eq.(6.6)), whereas in case of CHSH it is $\sqrt{2}$ and in Bell's original case it is $3 / 4$.

Although the violation of BI's is a manifestation of entanglement, as a criterion for separability it is rather poor. There exists a class of entangled states which satisfy the considered BI's, CHSH (6.18), Bell (6.24) but not our GBI (3.3) or (6.6). For a given entangled state there exists always some operator (entanglement witness) so that it satisfies the GBI for separable states but not for this entangled state. The class of these operators can be obtained by the positivity condition of Ref. [24]. On the other hand, as criterion for nonlocality the violation of the familiar BI's is of great importance.

Let us finally return again to the geometry of the quantum states (see also Ref. [35). For two spins there is a one parameter family of equivalence classes of pure states, interpolating between the separable one and the one containing $w_{\alpha=1}$. The latter is quite big and contains 4 orthogonal projections, the "Bell states". They are obtained by rotating $\vec{\sigma}_{A}$ by $180^{\circ}$ around each of the axis:

$$
\begin{aligned}
w_{\alpha=1} & =\frac{1}{4}\left(\mathbf{1}-\sigma_{A}^{x} \otimes \sigma_{B}^{x}-\sigma_{A}^{y} \otimes \sigma_{B}^{y}-\sigma_{A}^{z} \otimes \sigma_{B}^{z}\right)=: P_{0} \\
& \rightarrow \frac{1}{4}\left(\mathbf{1}-\sigma_{A}^{x} \otimes \sigma_{B}^{x}+\sigma_{A}^{y} \otimes \sigma_{B}^{y}+\sigma_{A}^{z} \otimes \sigma_{B}^{z}\right)=: P_{1} \\
& \rightarrow \frac{1}{4}\left(\mathbf{1}+\sigma_{A}^{x} \otimes \sigma_{B}^{x}-\sigma_{A}^{y} \otimes \sigma_{B}^{y}+\sigma_{A}^{z} \otimes \sigma_{B}^{z}\right)=: P_{2} \\
& \rightarrow \frac{1}{4}\left(\mathbf{1}+\sigma_{A}^{x} \otimes \sigma_{B}^{x}+\sigma_{A}^{y} \otimes \sigma_{B}^{y}-\sigma_{A}^{z} \otimes \sigma_{B}^{z}\right)=: P_{3} .
\end{aligned}
$$

However, there are far more since $\sigma_{A}$ and $\sigma_{B}$ can be rotated independently. 

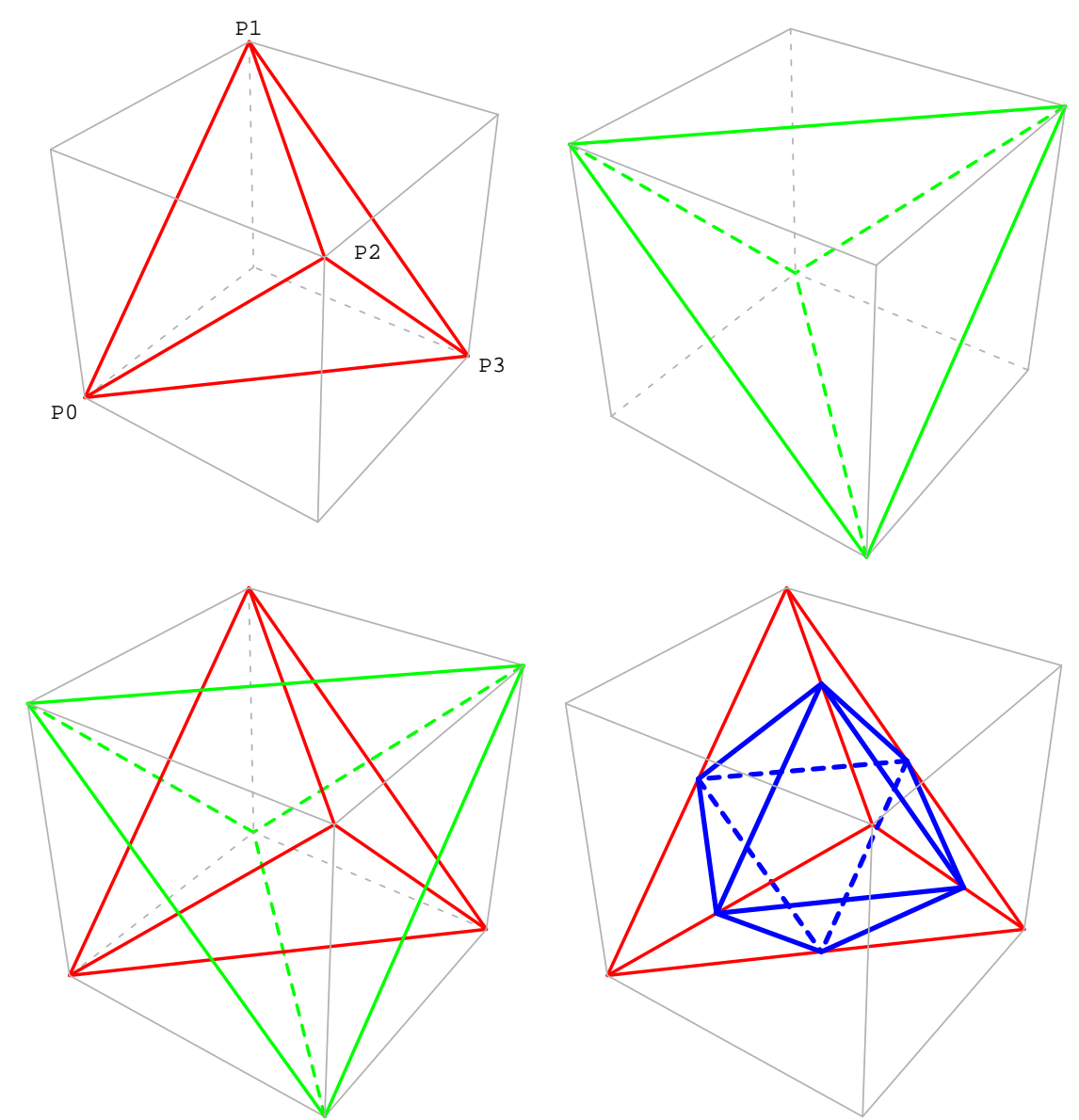

FIG. 3. In the left figure above we have plotted the tetrahedron of states described by the density matrix $w_{c}(6.29)$ in the $\vec{c}$-space and to the right the reflected set of states $\left(\mathbf{1}_{A} \otimes T_{B}\right) w_{c}$ is shown. In the left figure below we have plotted the intersection of the two sets $\left(w_{c}\right.$ and its mirror image) and, finally, to the right the double-pyramid of separable states $S \cap\left\{w_{c}\right\}$.

The matrix $c_{i j}$ in Eq.(2.5) will in general not be diagonalizible but by two independent orthogonal transformations on both spins it can be diagonalized. Thus the correlation part of a density matrix $w_{c}$ contains 3 parameters $c_{i}$ :

$$
w_{c}=\frac{1}{4}\left(\mathbf{1}+\sum_{i=1}^{3} c_{i} \sigma_{A}^{i} \otimes \sigma_{B}^{i}\right) .
$$

Density matrix $w_{c}$ can be expressed as convex combination of the projectors onto the 4 Bell states. Positivity requires that the $c_{i}$ are contained in the convex region spanned by the 4 points $(-1,-1,-1),(-1,1,1),(1,-1,1),(1,1,-1)$. This region is screwed and the intersection with its mirror image - compare with point vi) of the properties of $S$ characterizes the separable states $\sum_{i=1}^{3}\left|c_{i}\right| \leq 1$. Reflection in $c$-space is effected by time reversal on one spin and not on the other ("partial transposition") and the classically correlated states form the set invariant under this transformation. These properties are illustrated in Fig. 3 (see also Refs. [36, 37]). 
Finally, we would like to mention that the quantum states which are used in the model for decoherence of entangled systems in particle physics [38,39] also lie in the regions of the plotted separable and entangled states.

\section{SUMMARY AND CONCLUSION}

In this article we have used tangent functionals on the set of separable states as entanglement witnesses defining a generalized Bell inequality. The operators are vectors in the Hilbert space $\mathcal{H}_{s}$ with Hilbert Schmidt norm. We show that the euclidean distance of an entangled state to the separable states is equal to the maximal violation of the GBI with the tangent functional as entanglement witness. This description gives a nice geometric picture of separable and entangled states and their boundary, especially in the example of two spins. The advantage of considering the larger set of GBI's is that they are a criterion for separability (or entanglement) whereas the usual BI's are not.

\section{Acknowledgements:}

We are thankful to Katharina Durstberger for her drawings and to Fabio Benatti, Caslav Brukner, Franz Embacher, Walter Grimus, Beatrix Hiesmayr and Anton Zeilinger for fruitful discussions. We also thank Frank Verstraete and Jens Eisert for useful comments. The research was performed within the FWF Project No. P14143-PHY of the Austrian Science Foundation. 


\section{REFERENCES}

[1] E. Schrödinger, Naturwissenschaften 23, 807 (1935); 23, 823 (1935); 23, 844 (1935).

[2] A. Einstein, B. Podolsky and N. Rosen, Phys. Rev. 47, 777 (1935).

[3] A.K. Ekert, Phys. Rev. Lett. 67, 661 (1991).

[4] D. Deutsch and A.K. Ekert, Physics World Vol.11, No.3, p.47 (1998).

[5] R.J. Hughes, Contemp. Phys. 36, 149 (1995).

[6] W. Tittel, G. Ribordy and N. Gisin, Physics World Vol.11, No.3, p.41 (1998); W. Tittel, J. Brendel, H. Zbinden and N. Gisin, Phys. Rev. Lett. 81, 3563 (1998).

[7] C.H. Bennett, G. Brassard, C. Crépeau, R. Jozsa, A. Peres and W.K. Wootters, Phys. Rev. Lett. 70, 1895 (1993).

[8] J.-W. Pan, D. Bouwmeester, H. Weinfurter and A. Zeilinger, Nature 390, 575 (1997).

[9] A. Zeilinger, Physics World Vol. 11, No.3, 35 (1998).

[10] D. Bouwmeester, A. Ekert and A. Zeilinger (eds.), The physics of quantum information: quantum cryptography, quantum teleportation, quantum computations, Springer Verlag, Berlin, 2000.

[11] J.S. Bell, Speakable and Unspeakable in Quantum Mechanics, Cambridge University Press, 1987.

[12] M. Horodecki, P. Horodecki, R. Horodecki, Mixed state entanglement and quantum communication, in: Quantum information, G. Alber etal. (eds.), p. 151, Springer Tracts in Modern Physics 173, Springer Verlag Berlin, 2001.

[13] B.M. Terhal, Detecting quantum entanglement, quant-ph/0101032.

[14] C.H. Bennett, D.P. Di Vincenzo, J.A. Smolin, W.K. Wootters, Phys. Rev. A 54, 3824 (1996).

[15] A.S. Holevo, Problems of Int. Transm. 5, 247 (1979).

[16] V. Vedral, M.B. Plenio, M.A. Rippin, P.L. Knight, Phys. Rev. Lett. 78, 2275 (1997).

[17] O. Rudolph, A new class of entanglement measures, math-ph/0005011.

[18] H. Narnhofer, Entanglement, split and nuclearity in quantum field theory, preprint University of Vienna UWThPh-2001-25, to be published in Rep. Math. Phys.

[19] M. Horodecki, P. Horodecki, R. Horodecki, Phys. Rev. Lett. 84, 2014 (2000).

[20] C. Witte, M. Trucks, Phys. Lett. A 257, 14 (1999).

[21] M. Ozawa, Phys. Lett. A 268, 158 (2000).

[22] A. Uhlmann, Endlich dimensionale Dichtematrizen I, Wiss. Z. Karl-Marx-Univ. Leipzig 21, 421 (1972); II, ibid 22, 139 (1973).

[23] E.M. Henley, W. Thirring, Elementary quantum field theory, McGraw Hill, New York, 1962.

[24] M. Horodecki, P. Horodecki, R. Horodecki, Phys. Lett. A 223, 1 (1996).

[25] M.B. Terhal, Phys. Lett. A 271, 319 (2000), and quant-ph/9911057.

[26] J.F. Clauser, M.A. Horne, A. Shimony, R.A. Holt, Phys. Rev. Lett. 23, 880 (1969).

[27] A.O. Pittenger, M.H. Rubin, Lin. Alg. Appl. 346, 75 (2002).

[28] P. Hayden, B.M. Terhal, A. Uhlmann, On the LOCC classification of bipartite density matrices, quant-ph/0011095.

[29] S. Hill, W.K. Wootters, Phys. Rev. Lett. 78, 5022 (1997).

[30] F. Benatti, H. Narnhofer, A. Uhlmann, Rep. Math. Phys. 38, 123 (1996). 
[31] R.F. Werner, Phys. Rev. A 40, 4277 (1989).

[32] R.F. Werner, M.M. Wolf, Bell inequalities and entanglement, quant-ph/0107093.

[33] N. Gisin, Phys. Lett. A 154, 201 (1991).

[34] J.S. Bell, Physics 1, 195 (1964).

[35] F. Verstraete, J. Dehaene, B. De Moor, On the geometry of entangled states, quantph/0107155.

[36] R. Horodecki, M. Horodecki, Phys. Rev. A 54, 1838 (1996).

[37] K.G.H. Vollbrecht, R.F. Werner, Entanglement measures under symmetry, quant$\mathrm{ph} / 0010095$.

[38] R.A. Bertlmann, W. Grimus, B.C. Hiesmayr, Phys. Rev. D 60, 114032 (1999).

[39] R.A. Bertlmann, W. Grimus, Phys. Rev. D 64, 056004 (2001). 